\title{
Simulation modeling approaches to answer clinically relevant questions in breast cancer low-risk populations
}

\author{
Ana Calin ${ }^{1}$, Miguel Martin ${ }^{2}$, Sara Lopez-Tarruella ${ }^{2}$ \\ ${ }^{1}$ Radiation Oncology Service, Hospital General Universitario Gregorio Marañón, Madrid, Spain; ${ }^{2}$ Medical Oncology Service, Hospital General \\ Universitario Gregorio Marañón, Instituto de Investigación Sanitaria Gregorio Marañón (IiSGM), Universidad Complutense, CiberOnc, GEICAM, \\ Madrid, Spain \\ Correspondence to: Sara Lopez-Tarruella, MD, PhD. Medical Oncology Service, Hospital General Universitario Gregorio Marañón, Instituto de \\ Investigación Sanitaria Gregorio Marañón (IiSGM), Universidad Complutense, Maiquez nº7, Madrid 28007, Spain. Email: slopeztarruella@gmail.com. \\ Comment on: Jayasekera J, Li Y, Schechter CB, et al. Simulation Modeling of Cancer Clinical Trials: Application to Omitting Radiotherapy in Low-risk \\ Breast Cancer. J Natl Cancer Inst 2018. [Epub ahead of print].
}

Submitted Oct 20, 2018. Accepted for publication Oct 29, 2018.

doi: $10.21037 /$ atm.2018.10.68

View this article at: http://dx.doi.org/10.21037/atm.2018.10.68

Randomized controlled trials (RCTs) have been considered the gold standard required to address the value of a new intervention, in terms of efficacy and safety, by comparing it with the standard of care (control), and therefore generate the highest level of evidence (1). The regulatory authorities adopted RCTs as a requirement for new drug approvals, and the drug development process in oncology has been particularly fruitful in the past years although there is plenty of space for improving the success rate of a drug and accelerate the process. RCTs are essential in the drug registration process but the real evidence of a drug clinical utility needs to be adapted to the realworld scenario in which patients do not mimic the exact conditions of those included in the RCT. The magnitude of the effect demonstrated in RCTs is also highly variable, and the gap between statistically significant and clinically relevant differences has to be contextualized from a clinical and financial perspective. Besides efficacy, the full safety profile of a given drug or intervention is just partially covered in a single RCT and needs to be explored in wider populations and during longer periods to be completely defined (2). Hence, there are different priorities in clinical cancer research depending on the stakeholder. From the industry perspective, the acceleration of the process and the reduction of the developmental costs are top priorities. From the healthcare provider point of view, the affordability of cancer drugs is a major concern and the financial toxicity has to be addressed to guarantee the access to cancer treatments. However, academic research is focused on optimizing the use of new treatments far from merely commercial interests and registration trials. The definition of the role of a new drug in the context of multidisciplinary strategies or potential combinations is part of the academia's goals. But the funding for this independent initiatives and the harmonization of all the institutions, cooperative groups and regulatory authorities involved are quite challenging (3). And finally, and more importantly, patients are really concerned about the extrapolation of RCTs results to their particular situations, and how these results could help them to select the more suitable and balanced choice.

In parallel with the paradigm shift in the understanding of cancer biology (4), that has unraveled the multidimensional heterogeneity of cancer, other disciplines such as informatics, statistics or mathematics have also evolved and have been applied to cancer research, the advances in computational analysis are a key node in the precision oncology workflow. In this context emerges modeling and simulation $(M \& S)$, in a sense a substitute of physical experimentation, that builds a virtual mathematical model of a given physical model and calculates different results by changing the conditions (simulation) avoiding actual experimentations. $M \& S$ can be applied to diverse fields such as economics, manufacturing, or engineering but also to health sciences (5). M\&S offers the opportunity to test different biological scenarios in a virtual patient population by incorporating data from the different available sources 
and therefore help investigators in the decision makingprocess. $M \& S$ can be applied by pharmaceutical companies to speed up the drug development process and optimize the costs, but another application of this methodology is to address clinically relevant questions that are being debated by the scientific community and that impact cancer management strategies. CISNET (Cancer Intervention and Surveillance Modeling Network) is a consortium of NCIsponsored investigators that join efforts to apply $M \& S$ to important cancer research questions (6). Jayasekera et al. (7) have recently published a valuable practical example of their combined efforts to answer a highly relevant topic for early breast cancer treatment management, i.e., the consequences of omitting radiation therapy in low-risk breast cancer.

Precision oncology does not only consist on licensing new targeted drugs but also to optimize the use of the available treatment choices for individual patients. Early breast cancer treatment is moving from a traditional "addon" strategy to a more complex landscape where some patients with aggressive tumors need to escalate and other with low-risk BC can de-escalate treatments avoiding unnecessary toxicity while controlling disease. Molecular profiling has provided useful tools to adjust the risk of recurrence of BC patients, genomic platforms are now successfully used in the clinical practice to decide about the appropriateness of adding chemotherapy to endocrine therapy in hormone receptor-positive HER2-negative nodenegative early BC patients. Two important trials have been designed to test the clinical utility of genomic platforms in early breast cancer and recently achieved their respective goals [MINDACT (8) and TAILORx (9)]. Practical recommendations regarding de-escalation of therapy are a top priority nowadays as we can translate from the most recent St. Gallen International Consensus Conference (10).

The CISNET consortium uses a M\&S approach to evaluate the feasibility and probability of success of a clinical trial academic proposal, by NRG Oncology, aimed to answer the potential for the omission of radiotherapy (RT) in low-risk breast cancer patients defined by Oncotype score together with clinical variables (7). To conduct a trial in a low-risk population the large sample size required, the low event rates expected, and the long follow-up period needed to capture these events are potential barriers to consider in the academic setting that accounts with limited resources. Some aspects merit especial consideration in the CISNET M\&S approach. The source of data in model $M$ come from the EBCTCG Oxford meta-analysis of individual patient data from 17 randomized trials comparing
RT $v s$. no RT after breast conserving surgery (11), while in Model GE the data come from individual patient-level data from a low-risk patient subset of those trials (12). The use of data from meta-analysis has its limitations, as highlighted by the authors, insufficient information about such an important classic biomarker as the HER2 status, or about the excess of mortality associated to RT are examples of it. $M \& S$ helps in predicting outcomes, but available historical data sources have caveats that have to be considered when interpreting the results of the final prediction. The lowrisk definition adopted by the authors is based on classic clinical variables (tumor size of $2 \mathrm{~cm}$ or less, hormone receptor-positive/HER2-negative and no nodal involvement) and tumor biology, defined by an Oncotype Dx recurrence score of 18 or lower (equivalent to a $10 \%$ risk of distant relapse). This is a valid approach, but second-generation genomic test that compile clinical and biological variables could help to better identify low-risk patients in the clinical practice. In fact, the adequate biological identification of low-risk patients is far from being fully standardized and there are currently different attempts ongoing in this particular setting (13-15). There are some trials evaluating the omission of whole breast irradiation (WBI) in patients at low-risk like PRECISION (Profiling Early Breast Cancer for Radiotherapy Omission) (13). This is a non-randomized phase II trial, that uses Prosigna to predict 5-year locoregional recurrence rate in the ipsilateral breast or regional lymph-node. Patients in the low-risk group by Prosigna will omit WBI and will only receive endocrine therapy $v s$. those in the intermediate or high-risk that will be treated with WBI too. The EXPERT (Examining PErsonalised Radiation Therapy for Low-risk Early Breast Cancer) (14) is a randomized prospective phase III trial that is also using Prosigna to identify if low-risk patients could safely omit RT, with the ipsilateral breast tumor recurrence rate again as primary endpoint. Finally, the PRIMETIME trial (15), is a prospective biomarker-directed case-cohort study targeting women aged $\geq 60$, with $\mathrm{T} 1 \mathrm{~N} 0 \mathrm{M} 0$, hormone receptorpositive, HER2-negative, grade 1 or 2 tumors and with central testing of $\mathrm{Ki}-67$, that after breast conservative surgery will be scored according to IHC4+C marker to identify 'very low risk' patients that will not receive RT but will be treated with endocrine therapy as per standard of care.

Another aspect to consider in the $M \& S$ approach are the characteristics of the treatments used in the population included in both models, the RT techniques, or systemic treatments used (drugs, duration and combinations) in the patients included in the meta-analysis are not exactly the 
same as the ones offered today. And more importantly, the endpoint selection, that is crucial in this kind of deescalation trials. The authors selected the recurrencefree interval (RFI), that included loco-regional and distant recurrence besides breast cancer death, based on relative HR and not absolute risk differences. The noninferiority boundary would be established if the two-sided $90 \%$ confidence interval of RFI hazard ratio (RT vs. no-RT) was less than 1.7. It is important to consider that RT benefit in low-risk patients acts by reducing loco-regional recurrences, therefore the two outcomes included in the concept of RFI are not balanced in this particular population. With all these aspects in mind only $18.0 \%$ of the simulations in model GE and $3.7 \%$ in model $M$ concluded the non-inferiority for the omission of RT. To contextualize this prediction, it is also relevant to highlight that to make balanced decisions in the clinical practice we have to focus on the expected benefits derived from a given intervention but equally important is to consider the adverse event rate and toxicity profile of the same intervention. None of the models have included this information and this can be a limitation of this $M \& S$ approach to the local treatment de-escalation question.

The indication of locoregional treatment is currently based on classical risk factors including tumor size, age, nuclear grade, axillary lymph node involvement and traditional markers such estrogen receptor, HER2 expression and cell proliferation (ki-67). Molecular classification of BC suggests that the luminal A subtype has the lowest risk for local-regional recurrence and therefore this is the subgroup of interest to explore deescalation of RT. Advances in gene expression profiling and molecular tumor markers $(16,17)$ should be used to select patients for de-escalating RT. There are several options for decreasing the burden of RT such as the total dose reduction by omitting the boost, decreasing the target volume of the breast irradiation by using accelerated partial breast irradiation (APBI), and using hypofractionated RT schedules (lowering treatment time from 5 to 3 weeks). On the other hand, modern RT tools can increase the therapeutic ratio (i.e., a deep inspiration breath hold to reduce cardiopulmonary exposure and image guidance to increase the accuracy of treatment delivery). In the pooled analysis, Jayasekera's (12) definition of low-risk BC patients overlaps with the suitable group defined by ASTRO to select patients for APBI (18). APBI has been introduced as an alternative treatment method to WBI for selected patients with low-risk BC. Potential advantages include shorter time, even with intraoperative techniques in 1 day, improved cosmesis and decrease doses in organs at risks like lung or heart and therefore decrease the potential harms of the RT. Late radiation toxicity with intraoperative RT is significantly lower than in the WBI group (19). Minimizing treatment to warrant a good quality of life without compromising survival is relevant in the low-risk BC group, particularly for elderly patients. Thus, in the clinical decision algorithm about the indication of RT in this type of BC patients, we have to incorporate APBI as a valid treatment choice adapted for low-risk patients in terms of less potential harms and equivalent overall survival and mortality rates (20).

All models have to be then interpreted as virtual simulations that can help to highlight particular limitations of the original study design, but they are not intended to act as replacements of real clinical trials to answer the questions as the ones summarized above. Jayasekera's work (7) led to the conclusion that the trial on its original design was unlikely to demonstrate the non-inferiority of the omission of RT in low-risk patients. However, the potential of de-escalation of radiation therapy does not end with this approach, and we could adapt the proposal to include new RT techniques that offer better tolerability while maintaining a potential protective effect. Adapted risk RT is the future as well as we are adapting endocrine therapy duration and chemotherapy regimens for early breast cancer patients. In that way, RCT are necessary but difficult to do, so tools like M\&S may help us to design and conduct them.

\section{Acknowledgements}

None.

\section{Footnote}

Conflicts of Interest: M Martin has received speakers honoraria or honoraria for participation in Advisory Boards from AstraZeneca, Novartis, Roche-Genentech, Pfizer Glaxo, Pharmamar, Taiho Oncology, and Lilly, and research grants from Novartis and Roche; S Lopez-Tarruella has received speakers honoraria, travel grants or honoraria for participation in Advisory Boards from AstraZeneca, Novartis, Roche, Pfizer, Celgene, Pierre-Fabre, and Eisai. A Calin has no conflicts of interest to declare.

\section{References}

1. Bothwell LE, Podolsky SH. The Emergence of 
the Randomized, Controlled Trial. N Engl J Med 2016;375:501-4.

2. Booth CM, Tannock IF. Randomised controlled trials and population-based observational research: partners in the evolution of medical evidence. Br J Cancer 2014;110:551-5.

3. Negrouk A, Lacombe D, Cardoso F, et al. Safeguarding the future of independent, academic clinical cancer research in Europe for the benefit of patients. ESMO Open 2017;2:e00187.

4. Jürgensmeier JM, Eder JP, Herbst RS. New strategies in personalized medicine for solid tumors: molecular markers and clinical trial designs. Clin Cancer Res 2014;20:4425-35.

5. Kimko H, Pinheiro J. Model-based clinical drug development in the past, present and future: a commentary. Br J Clin Pharmacol 2015;79:108-16.

6. Alagoz O, Berry DA, de Koning HJ, et al. Introduction to the Cancer Intervention and Surveillance Modeling Network (CISNET) Breast Cancer Models. Med Decis Making 2018;38:3S-8S.

7. Jayasekera J, Li Y, Schechter CB, et al. Simulation Modeling of Cancer Clinical Trials: Application to Omitting Radiotherapy in Low-risk Breast Cancer. J Natl Cancer Inst 2018. [Epub ahead of print].

8. Cardoso F, van't Veer LJ, Bogaerts J, et al. 70-Gene Signature as an Aid to Treatment Decisions in Early-Stage Breast Cancer. N Engl J Med 2016;375:717-29.

9. Sparano JA, Gray RJ, Makower DF, et al. Adjuvant Chemotherapy Guided by a 21-Gene Expression Assay in Breast Cancer. N Engl J Med 2018;379:111-21.

10. Curigliano G, Burstein HJ, P Winer E, et al. De-escalating and escalating treatments for early-stage breast cancer: the St. Gallen International Expert Consensus Conference on the Primary Therapy of Early Breast Cancer 2017. Ann Oncol 2017;28:1700-12.

11. Darby S, McGale P, Correa C, et al. Effect of radiotherapy after breast-conserving surgery on 10-year recurrence and 15-year breast cancer death: meta-analysis of individual

Cite this article as: Calin A, Martin M, Lopez-Tarruella S. Simulation modeling approaches to answer clinically relevant questions in breast cancer low-risk populations. Ann Transl Med 2018;6(Suppl 1):S80. doi: 10.21037/atm.2018.10.68 patient data for 10,801 women in 17 randomised trials. Lancet 2011;378:1707-16.

12. Jayasekera J, Schechter CB, Sparano JA, et al. Effects of Radiotherapy in Early-Stage, Low-Recurrence Risk, Hormone-Sensitive Breast Cancer. J Natl Cancer Inst 2018. [Epub ahead of print].

13. The PRECISION Trial (Profiling Early Breast Cancer for Radiotherapy Omission): A Phase II Study of BreastConserving Surgery Without Adjuvant Radiotherapy for Favorable-Risk Breast Cancer. Available online: https:// clinicaltrials.gov/ct2/show/NCT02653755

14. EXamining PErsonalised Radiation Therapy for Low-risk Early Breast Cancer (EXPERT). Available online: https:// clinicaltrials.gov/ct2/show/NCT02889874

15. Kirwan CC, Coles CE, Bliss J, et al. It's PRIMETIME. Postoperative Avoidance of Radiotherapy: Biomarker Selection of Women at Very Low Risk of Local Recurrence. Clin Oncol (R Coll Radiol) 2016;28:594-6.

16. Jegadeesh NK, Kim S, Prabhu RS, et al. The 21-gene recurrence score and locoregional recurrence in breast cancer patients. Ann Surg Oncol 2015;22:1088-94.

17. Solin LJ, Gray R, Goldstein LJ, et al. Prognostic value of biologic subtype and the 21-gene recurrence score relative to local recurrence after breast conservation treatment with radiation for early stage breast carcinoma: results from the Eastern Cooperative Oncology Group E2197 study. Breast Cancer Res Treat 2012;134:683-92.

18. Correa C, Harris EE, Leonardi MC, et al. Accelerated Partial Breast Irradiation: Executive summary for the update of an ASTRO Evidence-Based Consensus Statement. Pract Radiat Oncol 2017;7:73-9.

19. Sperk E, Welzel G, Keller A, et al. Late radiation toxicity after intraoperative radiotherapy (IORT) for breast cancer: results from the randomized phase III trial TARGIT A. Breast Cancer Res Treat 2012;135:253-60.

20. Marta GN, Macedo CR, Carvalho Hde A, et al. Accelerated partial irradiation for breast cancer: systematic review and meta-analysis of 8653 women in eight randomized trials. Radiother Oncol 2015;114:42-9. 